\title{
GEO-REFERENCED SPATIAL MULTIMEDIA APPLICATION FOR AGRICULTURAL RESOURCE MANAGEMENT
}

\author{
Hui Liu ${ }^{1}$, Gang Liu ${ }^{1}$, Zhijun Meng ${ }^{2}$ \\ ${ }^{1}$ Key Laboratory of Modern Precision Agriculture System Integration Research, Ministry of \\ Education, China Agricultural University, Beijing 100083, China Corresponding author, \\ Email:pac@cau.edu.cn \\ ${ }^{2}$ National Engineering Research Center for Information Technology in Agriculture, Beijing, \\ P. R. China
}

Abstract: Agricultural resource management is one of important technologies in agricultural application. The new technology, geo-referenced spatial multimedia has revolutionized the data collection, storage, management and display for agricultural resource management. This paper presents geo-referenced spatial multimedia technology including the system architecture, data collection procedures and data processing based on VMS system of Red Hen Company, and then introduced the application for agricultural resource management.

Keywords: GPS, GIS, Geo-referenced spatial multimedia, precision agriculture

\section{INTRODUCTION}

In recent years, more and more information technologies such as Global Positioning Systems (GPS) and Geographic Information Systems (GIS) are applied in agricultural resource management for digital data collection, storage, manipulation and display. Although many GIS have been successfully implemented, it has become quite clear that two-dimensional maps cannot precisely present multidimensional and dynamic spatial phenomena. Geo-referenced

Liu, H., Liu, G. and Meng, Z., 2008, in IFIP International Federation for Information Processing, Volume 259; Computer and Computing Technologies in Agriculture, Vol. 2; Daoliang Li; (Boston: Springer), pp. 1303-1306. 
spatial multimedia is a new technology that merges photography and geography to help users understand space by experiencing abstraction and reality through the integration of maps and images.

Some researchers have reported their applications of geo-referenced spatial multimedia. Ayers et al. (2000) used the video mapping system for field data collection in Lorry State Park, and generated geo-referenced information, which provided visual source and observation for management of the park in terms of timing. Liu et al. (2000) explored the application of the GPS/GIS and VMS in agricultural machinery.

This paper presents geo-referenced spatial multimedia technology based on video mapping systems (VMS) of Red Hen Company, and then introduces VMS applications in agricultural resource management.

\section{MATERIAL AND METHODS}

\subsection{Geo-referenced spatial multimedia technology}

Video mapping systems (VMS) developed by Red Hen Systems is a comprehensive spatial multimedia system including data collection devices and data processing software. System architecture can be described as threelayer framework - hardware layer, management layer and application layer.

The hardware layer is for data collections. In the first method, a camera or a video and a GPS receiver work separately, there is no cable between them. But it is necessary to log GPS track and take still image or video at the same time and same place. In another method, a specific device is used for data collections of merging for position and media to achieve data merging.

After data collection, these multi-resources data are imported to the management layer, MediaMapper, which is data processing software. MediaMapper software automatically merges these source media with spatial information from GPS receivers, building an interactive media map. MediaMapper can also export into GIS format and HTML file.

Based on the processed spatial media data, differential areas can design their application system according to differential needs such agricultural management, city facilities management and environment protection.

\subsection{Applications in agricultural resource management}

Many high technology of precision agriculture including GPS, GIS, ES, variable management have been applied in our demo farm. The interesting positions such as farm infrastructure, sampling points, pest occurrence could be imported and displayed in the digital map for precision management, 
whilst most of these positions were also photographed. But the interesting positions could not be related with their photos in the past. We require a means of associating GPS data with pictures and videos. Geo-referenced spatial multimedia mapping technology becomes the ideal selection. The objective of our application was to summarize the utilization of GPS, GIS and VMS to obtain geo-referenced video and images for agriculture resource management, and to export the spatial data into ArcGIS for analysis and management.

The image or video and their locations for agricultural resource data had been collected in two ways of post-processing integration and real-time merging.

A Nicon digital camera and a Garmin portable GPS receiver were selected for data collection of post-processing integration. It was necessary to calibrate time of camera and GPS receiver before collection. The simplest way for time calibration was taking photo for UTC time display of the receiver with the camera. If using video camera, a video for UTC time display could be taken for time calibration. It is sure to make the photo or video for time calibration distinguishable because of it is important for data processing. GPS receiver was configured to output GGA, RMC sentences. Then data collection was beginning. Taking photos at the interesting location while logging GPS track. It is sure to keep the same step while operating the camera and receiver for data matching during processing.

In real-time merging method, a Sony digital video camera and a VMS device which had inner GPS module for positioning were selected. It was necessary to make sure to connect devices correctly. The video camera and the VMS device were connected using the microphone (MIC) connector. After the antenna of GPS module connected and the satellites locked, it was time to take video of agricultural resource data when recording real-time NMEA GGA and RMC strings on the videotape.

\subsection{Results}

GPS receiver, digital camera, video camera and VMS device were connected the computer separately for data input. MediaMapper provided a data import wizard, which made the operation easier. After data processed, users could add the layers of background digital map and set the options for display resulted in a geo-referenced spatial multimedia map.

The data of agricultural application were exported into ArcGIS of ESRI Company in SHP file for management and analysis after the extension tool setup for the ArcGIS environment. Figure 1 shows a sprinkler of the farm in ArcView. The data were also created and exported in HTML format. Figure 2 shows a HTML file. We put HTML files onto the HTTP server system and provided consultation via Internet. 


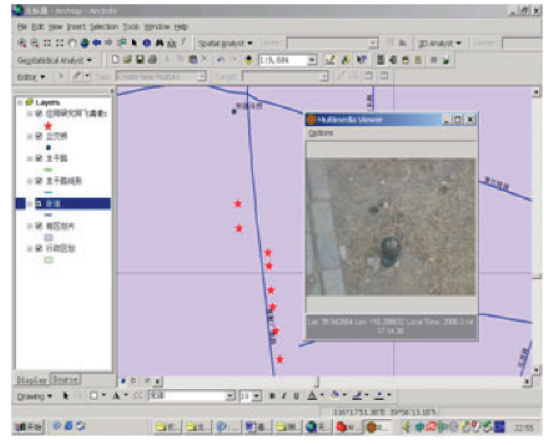

Figure 1. ArcView display of spatial multimedia data

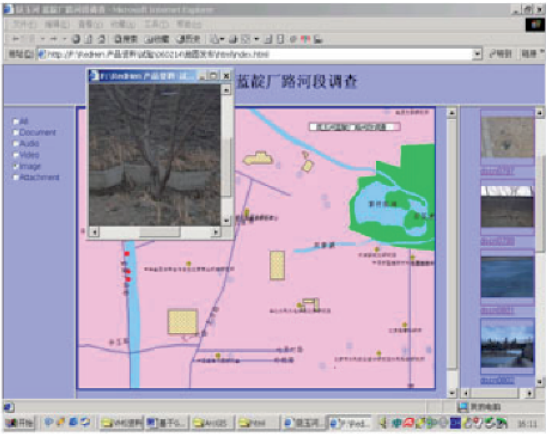

Figure 2. HTML files of spatial multimedia data

\section{CONCLUSION}

In agriculture, geo-referenced spatial multimedia could be applied not only in resource management such as geo-referenced data collection in fields, machines and facilities management in farms, but also in crop analysis, pest and disease prevention and weeds monitoring. This new technology should highly improve visual display and management efficiency in agricultural activities, and help people to find enjoyment during farming.

\section{REFERENCES}

Ayers, P., Juhua Liu, and J. Hocheder. GPS-based video mapping for nature resource management. Proceedings of the Second International Conference on Geospatial Information in Agricultural and Forestry. pp. I-464-468. Disney's Coronado Springs Resort, Lake Buena Vista, Florida. January 10-12, 2000.

Liu, Juhua, P. Ayers, and N. Geng. Database Modeling of Agricultural Machinery Safety and Production Based on Integrating GPS/GIS and VMS Technology. Presented at 2000 ASAE Annual International Meeting Sponsored by ASAE Milwaukee, Wisconsin, USA July 9-12, 2000. 